\title{
The Luminosity Function of Galaxies in Some Nearby Clusters
}

\author{
Mariwan A. Rasheed ${ }^{1,2}$ and Khalid K. Mohammad ${ }^{2}$ \\ ${ }^{1}$ Development Center for Research and Training, University of Human Development, Sulaimani, Kurdistan Region, Iraq, \\ ${ }^{2}$ Department of Physics, College of Science, University of Sulaimani, Sulaimani, Kurdistan Region, Iraq
}

\section{A B S T R A C T}

In the present work, the galaxy luminosity function (LF) has been studied for a sample of seven clusters in the redshift range $(0.0 \lesssim z \lesssim 0.1)$, within Abell radius $\left(1.5 h^{-1} \mathrm{Mpc}\right)$, in the five SDSS passbands ugriz. In each case, the absolute magnitude distribution is found and then fitted with a Schechter function. The fitting is done, using the $\chi^{2}-$ minimization method to find the best values of Schechter parameters $\Phi^{*}$ (normalization constant), $M^{*}$ (characteristic absolute magnitude), and $\alpha$ (faint-end slope). No remarkable changes are found in the values of $M^{*}$ and $\alpha$, for any cluster, in any passband. Furthermore, the LF does not seem to vary with such cluster parameters as richness, velocity dispersion, and Bautz-Morgan morphology. Finally, it is found that $\mathrm{M}^{*}$ becomes brighter toward redder bands, whereas almost no variation is seen in the value of $\alpha$ with passband, being around $(-1.00)$.

Index Terms: Galaxies, Clusters, Luminosity function, Galaxy formation, Galaxy evolution

\section{INTRODUCTION}

Galaxies come in a diversity of sizes and cover a very wide range of luminosities, extending from the faintest dwarfs to the most luminous giant ellipticals. To know how these galaxies are distributed with respect to their luminosities, the luminosity function (LF) is used. It is one of the most important techniques used for studying galaxy formation and evolution. A suitable approximation to this function was given by Paul Schechter in 1976 [1]. It can be written as

$$
\Phi(L)=\left(\frac{\Phi^{*}}{L^{*}}\right)\left(\frac{L}{L^{*}}\right)^{\alpha} \exp \left(-\frac{L}{L^{*}}\right)
$$

\section{Access this article online}

DOI: 10.21928/uhdjst.v5n2y2021.pp1-10

E-ISSN: 2521-4217

P-ISSN: 2521-4209

Copyright (C) 2021 Al-Janabi, et al. This is an open access article distributed under the Creative Commons Attribution Non-Commercial No Derivatives License 4.0 (CC BY-NC-ND 4.0) where, $L^{*}$ is a characteristic luminosity, indicating the change from power law $\left(L<L^{*}\right)$ to exponential law $\left(L>L^{*}\right), \alpha$ is the faint-end slope, and $\Phi^{*}$ is a normalization constant for the distribution. These parameters may take different values for different morphological types and also for different environments. Considering an interval $d$ L in luminosity, $\Phi(L)$ $d$ L gives the number density of galaxies.

Galaxy clusters are ideal systems for studying the galaxy LF due to the existence of a large number of galaxies at almost the same distance. Many studies have thus been devoted to the LF of cluster galaxies to discover the influence of environment on their evolution. After the earlier works on the LF, carried out by Hubble (1936) [2], [3], Zwicky (1942) [4], Oemler (1974) [5], and others, Schechter (1976) [1] proposed the analytic expression given by Equation (1), which is called the Schechter function. He suggested that the cluster LF is universal in shape. This universality has been supported by various studies [6], [7], [8]. However, studies carried out by others [9], [10], [11] have demonstrated that the shape of the cluster LF is not universal.

Corresponding author's e-mail: Khalid K. Mohammad, Department of Physics, College of Science, University of Sulaimani, Sulaimani, Kurdistan Region, Iraq. E-mail: khalid.mohammad@univsul.edu.iq 
The LF of cluster galaxies has been compared to that of field galaxies through several studies. Some of these studies found them to be identical [12], [13], [14], while others found them to be different [8], [15], [16]. The cluster LF has been found to vary with cluster-centric radius [11], [17]. This is because different galaxy morphological types have different LFs [18] and that the mixture of these morphological types varies with cluster-centric radius, according to the morphology-density relation [19]. In fact, studying the variation of the cluster LF with such characteristics as cluster-centric radius, galaxy morphologies, and, also, galaxy colors is very important in constraining theories of galaxy formation and evolution.

In the present work, we study the LF of a sample of seven Abell-type galaxy clusters having redshifts in the range $(0.0$ $\lesssim z \lesssim 0.1$ ). A detailed description of the sample is given in Section 2, and the results and discussion are presented in Section 3. Our conclusions are outlined in Section 4. Throughout the work, $\Lambda$ CDM parameters $\left(\Omega_{\mathrm{M}}=0.27, \Omega_{\Lambda}\right.$ $=0.73, \mathrm{H}_{0}=73 \mathrm{~km} \mathrm{~s}^{-1} \mathrm{Mpc}^{-1}$ ) are used.

\section{SAMPLE AND DATA}

In this work, we consider a sample of seven nearby galaxy clusters, selected from Abell catalogue [20] within the redshift range $(0.0 \lesssim \mathrm{z} \lesssim 0.1)$. Their basic data are given in Table 1. All possible member galaxies within Abell radius $\left(R_{A}=1.5 \mathrm{~h}^{-1} \mathrm{Mpc}\right)$ of each cluster were taken into account. For membership confirmation, redshift data were obtained from the Sloan Digital Sky Survey (SDSS-DR9) [21] database (for A1656, A2199, and A2147) and the NASA/IPAC Extragalactic Database (NED) (for A2255 and A2144). For the other two clusters A85 and A2029, redshift data were

\section{TABLE 1: The basic data of the cluster sample.}

\begin{tabular}{|c|c|c|c|c|c|c|}
\hline \multirow[t]{2}{*}{ Cluster } & \multicolumn{2}{|c|}{ Equ. J2000.0 } & \multirow{2}{*}{ Redshift } & \multirow{2}{*}{$\begin{array}{l}\text { Velocity } \\
\text { dispersion } \\
\sigma(\mathrm{km} / \mathrm{s})\end{array}$} & \multirow{2}{*}{$\begin{array}{l}\text { Richness } \\
\text { class }\end{array}$} & \multirow{2}{*}{$\begin{array}{c}\text { Bautz- } \\
\text { Morgan } \\
\text { type }\end{array}$} \\
\hline & R.A. & Dec. & & & & \\
\hline A1656 & $\begin{array}{c}125 \\
948.7\end{array}$ & $+275,850$ & 0.0231 & 970 & 2 & II \\
\hline A2199 & $\begin{array}{c}162 \\
838.0\end{array}$ & $+393,255$ & 0.0302 & 733 & 2 & I \\
\hline A2147 & $\begin{array}{c}160 \\
218.7\end{array}$ & $+160,112$ & 0.0350 & 859 & 1 & III \\
\hline A0085 & $\begin{array}{c}004 \\
150.1\end{array}$ & $-091,809$ & 0.0551 & 963 & 1 & I \\
\hline A2029 & $\begin{array}{c}151 \\
055.0\end{array}$ & $+054,312$ & 0.0773 & 1247 & 2 & I \\
\hline A2255 & $\begin{array}{c}171, \\
231.0\end{array}$ & $+640,533$ & 0.0806 & 998 & 2 & II-III \\
\hline A2142 & $\begin{array}{c}155 \\
820.6\end{array}$ & $+271,337$ & 0.0909 & 1008 & 2 & II \\
\hline
\end{tabular}

obtained from Agulli et al. (2016) [22] and Sohn et al. (2017) [23], respectively. Petrosian magnitudes, taken from the SDSS database, were used for calculating the absolute magnitudes in the five bands $u(3551 \AA), g(4686 \AA), r(6166 \AA), i(7480 \AA)$, and $z$ (8932 $\AA$ ). These magnitudes were then corrected for galactic foreground extinction, using values given by Schlafly and Finkbeiner (2011) [24], and, also, K-corrected, using a method given by Chilingarian et al. 2010 [25] and Chilingarian and Zolotukhin (2012) [26].

With both of these corrections taken into consideration, the relation between absolute and apparent magnitudes for any passband can be written as:

$$
M=m-5 \log _{10}\left(D_{L}\right)-25-K(z)-A_{\lambda} / \sin (b)
$$

where, $D_{L}$ is the luminosity distance, $K(\vartheta)$ is the $\mathrm{K}$ correction, $A_{\lambda}$ is the galactic foreground extinction, and $b$ is the galactic latitude.

\section{RESULTS AND DISCUSSION}

It is convenient to write the LF in terms of absolute magnitude, $M$, rather than luminosity [27]. These two quantities are related through the expression

$$
M^{*}-M=2.5 \log \left(\frac{L}{L^{*}}\right)
$$

Hence, the LF becomes [28]

$$
\begin{aligned}
& \Phi(M)=(0.4 \ln 10) \Phi^{*} 10^{0.4(\alpha+1)\left(M^{*}-M\right)} \\
& * \exp \left(-10^{0.4\left(M^{*}-M\right)}\right)
\end{aligned}
$$

where, $M^{*}$ is the characteristic absolute magnitude corresponding to $L^{*}$.

Figures 1-5 show the absolute magnitude distributions of galaxies in the ugriz bands, within $R_{A}=1.5 \mathrm{~h}^{-1} \mathrm{Mpc}$, for the whole cluster sample, each fitted with a Schechter function. The fitting is done using the $\chi^{2}$ - minimization method, and for each case, we vary the magnitude bins until we get the best $\chi^{2}$ that gives the optimal values of Schechter parameters. Table 2 summarizes the results of the best-fitting Schechter parameters $\phi^{*}, \mathrm{M}^{*}$, and $\alpha$, for the whole clusters, in all passbands.

Since $\phi^{*}$ is just a normalization constant which defines the overall density of galaxies, we focus our attention only on 
Mariwan A. Rasheed and Khalid K. Mohammad: Luminosity Function of Galaxies

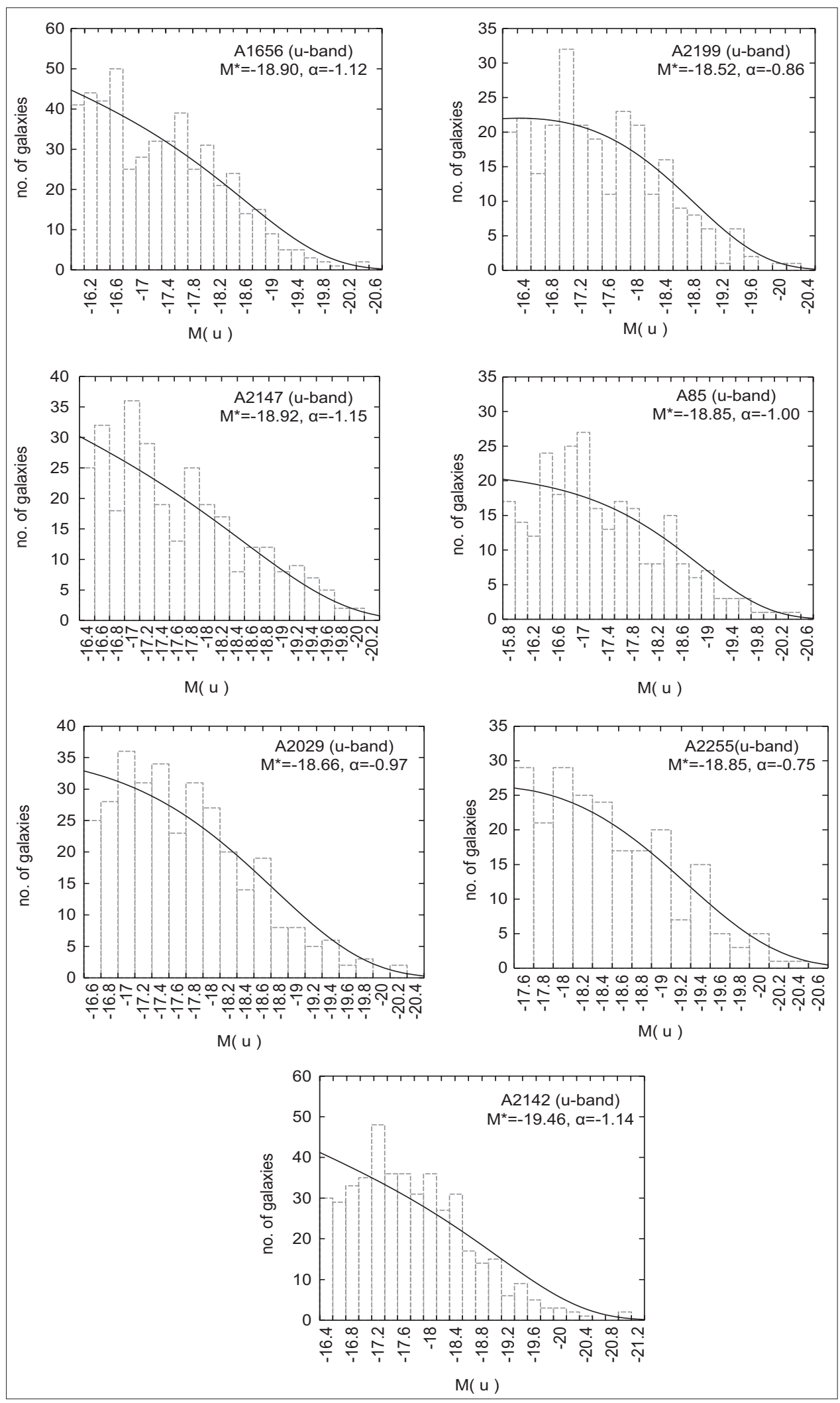

Fig. 1. The luminosity distributions (histograms) of the cluster sample in the u-band, fitted with Schechter functions (solid curves). 
Mariwan A. Rasheed and Khalid K. Mohammad: Luminosity Function of Galaxies
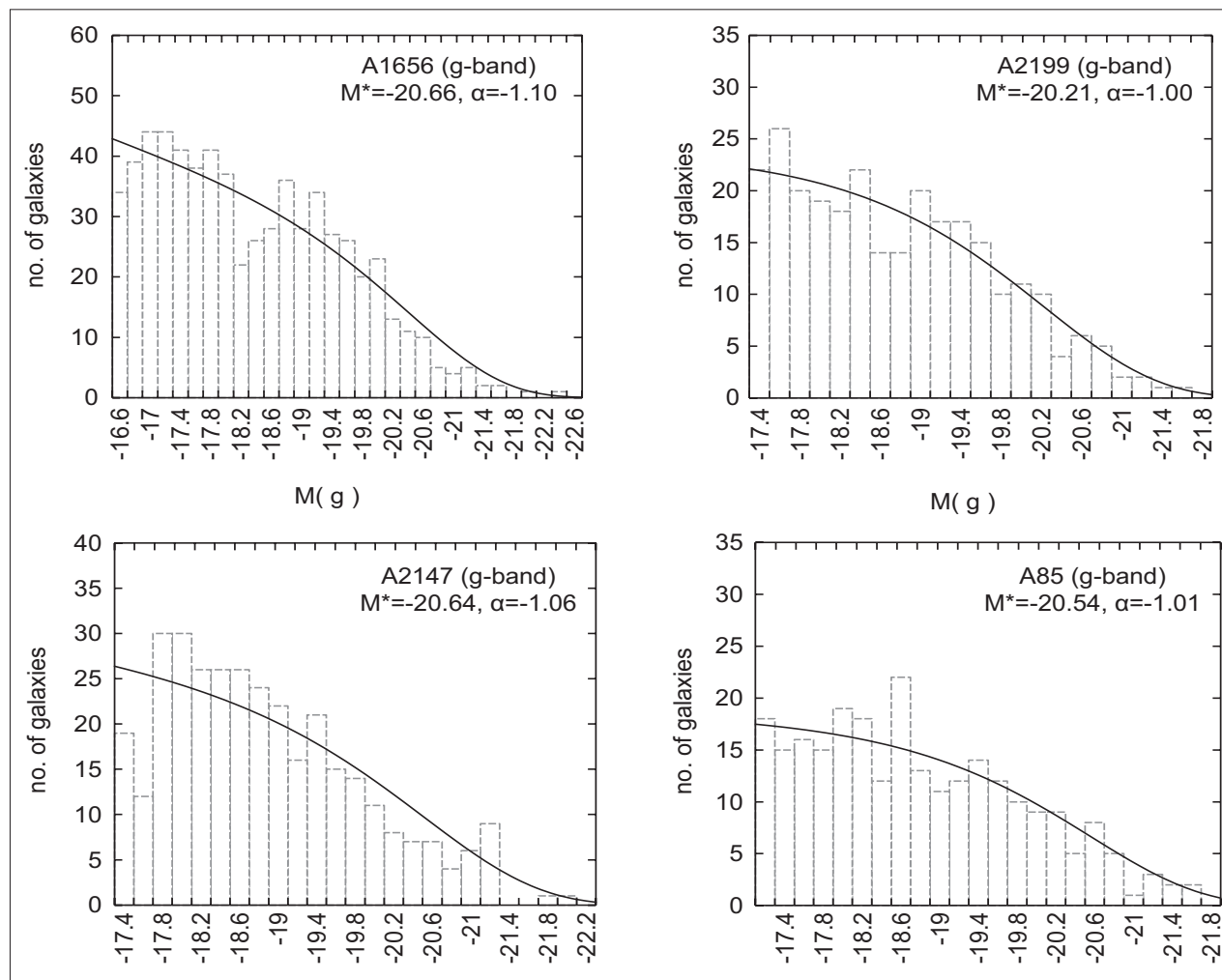

$\mathrm{M}(\mathrm{g})$
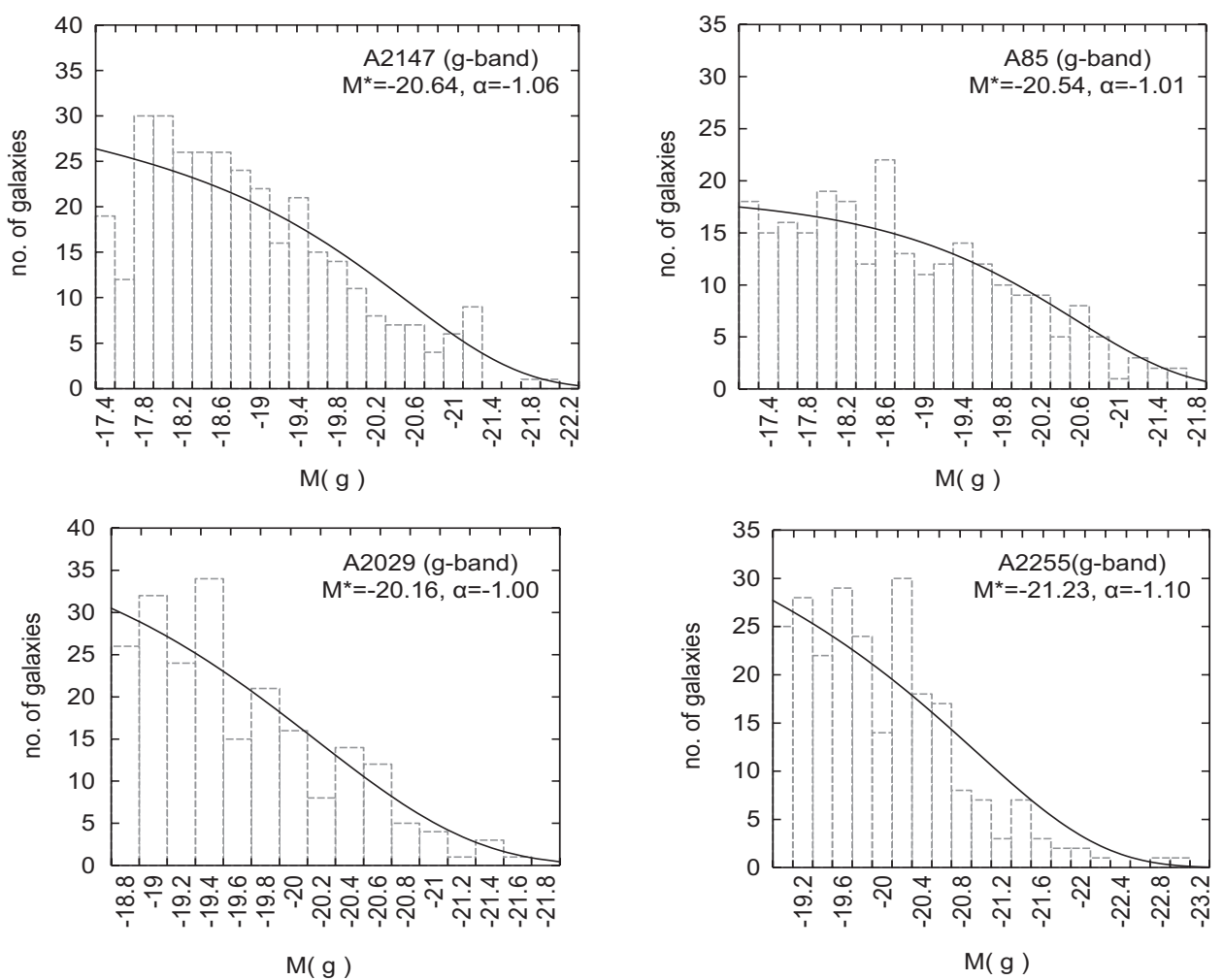

$\mathrm{M}(\mathrm{g})$

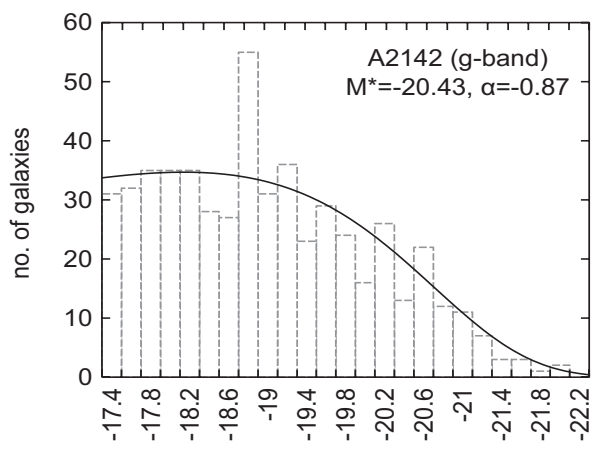

$\mathrm{M}(\mathrm{g})$

Fig. 2. The luminosity distributions (histograms) of the cluster sample in the g-band, fitted with Schechter functions (solid curves). 
Mariwan A. Rasheed and Khalid K. Mohammad: Luminosity Function of Galaxies
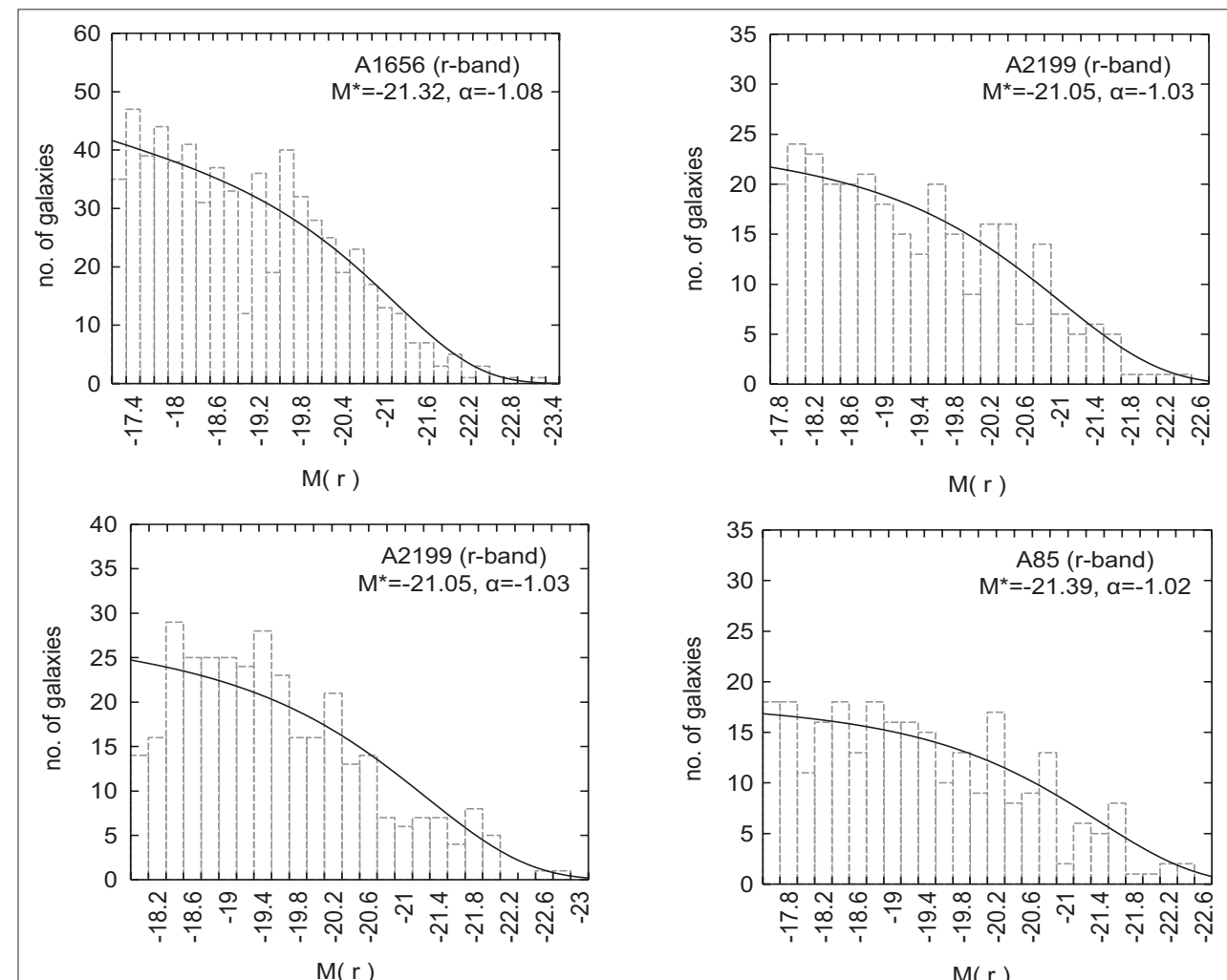

$M(r)$
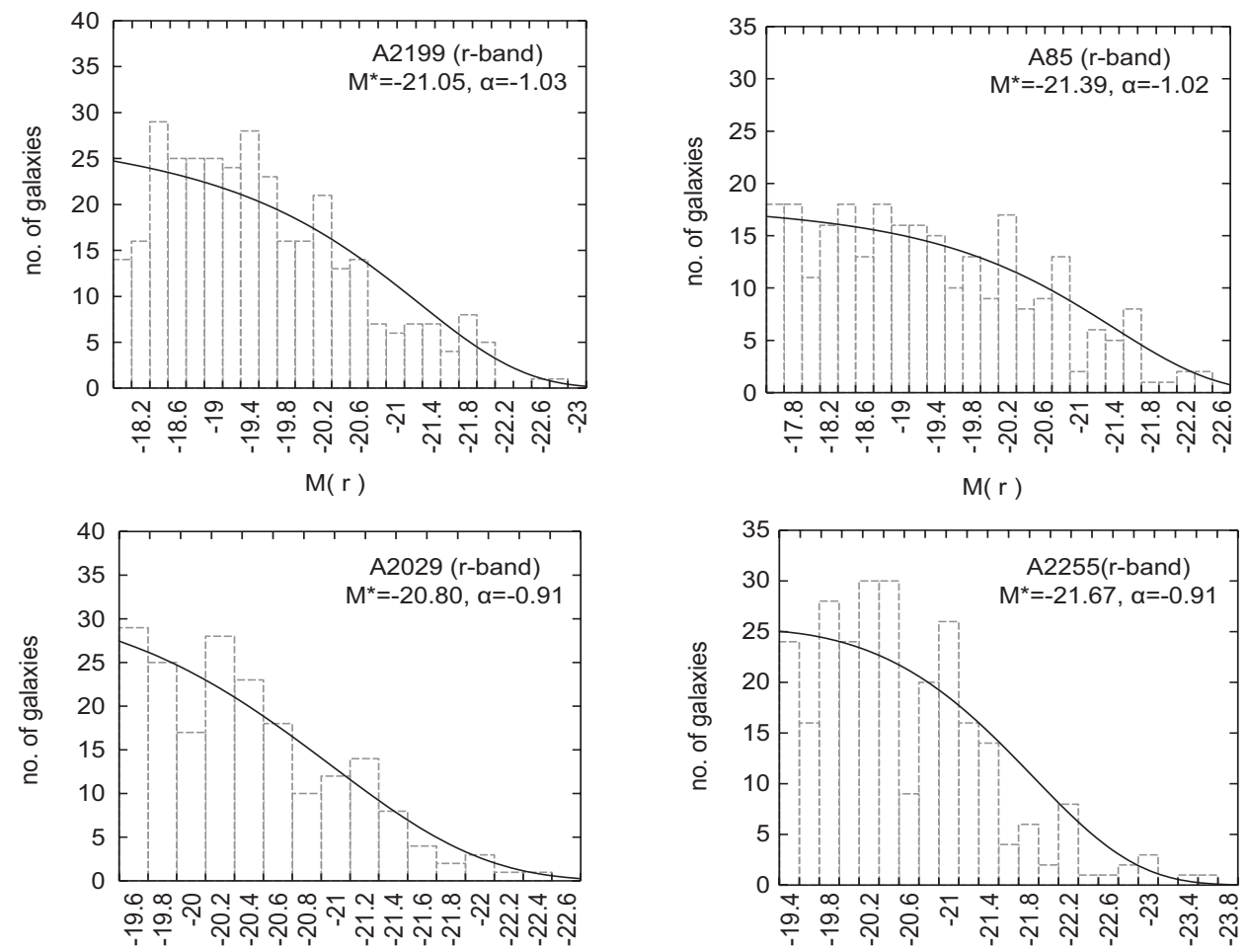

$M(r)$

$M(r)$

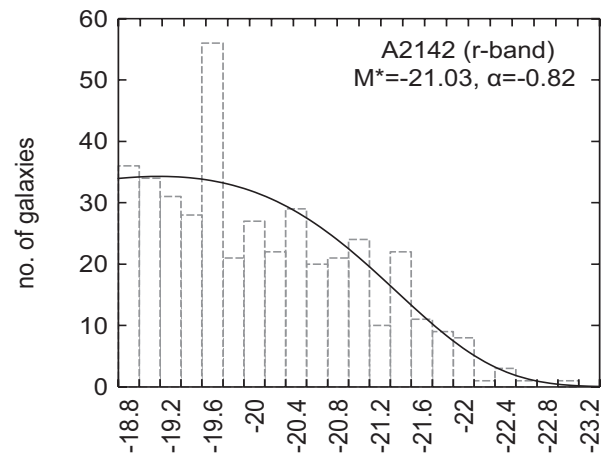

$M(r)$

Fig. 3. The luminosity distributions (histograms) of the cluster sample in the r-band, fitted with Schechter functions (solid curves). 
Mariwan A. Rasheed and Khalid K. Mohammad: Luminosity Function of Galaxies
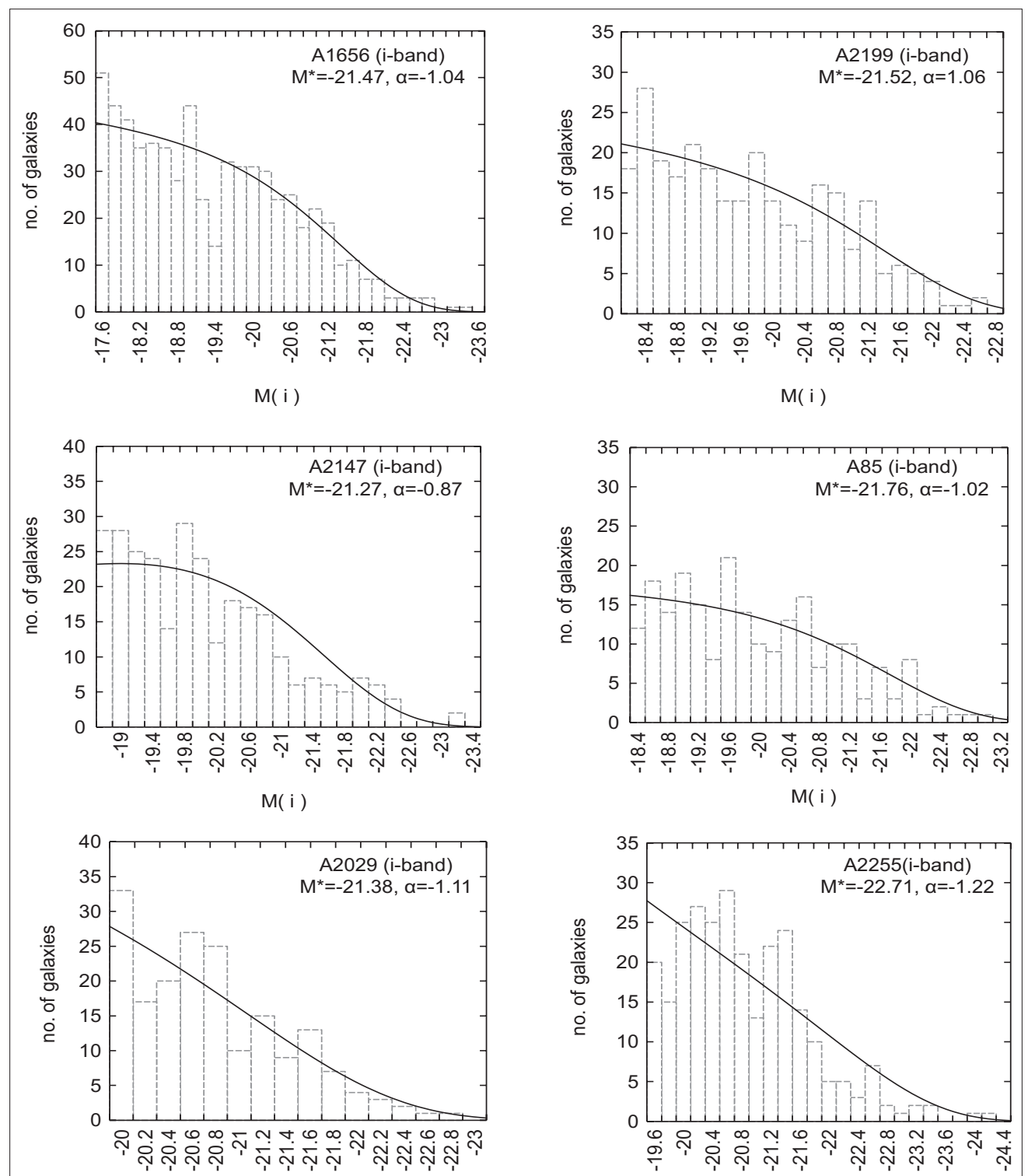

$M(\mathrm{i})$

$M($ i $)$

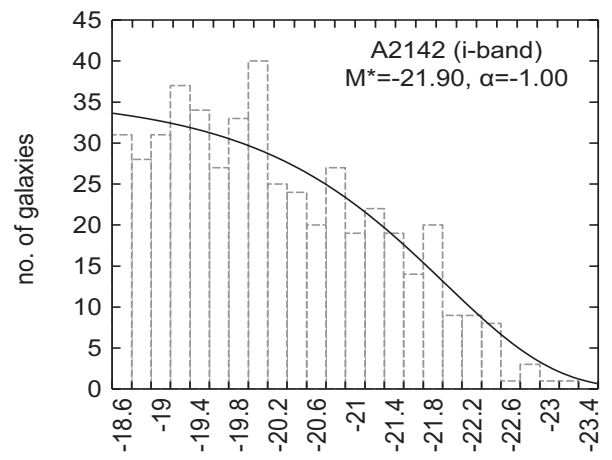

$M(\mathrm{i})$

Fig. 4. The luminosity distributions (histograms) of the cluster sample in the i-band, fitted with Schechter functions (solid curves). 

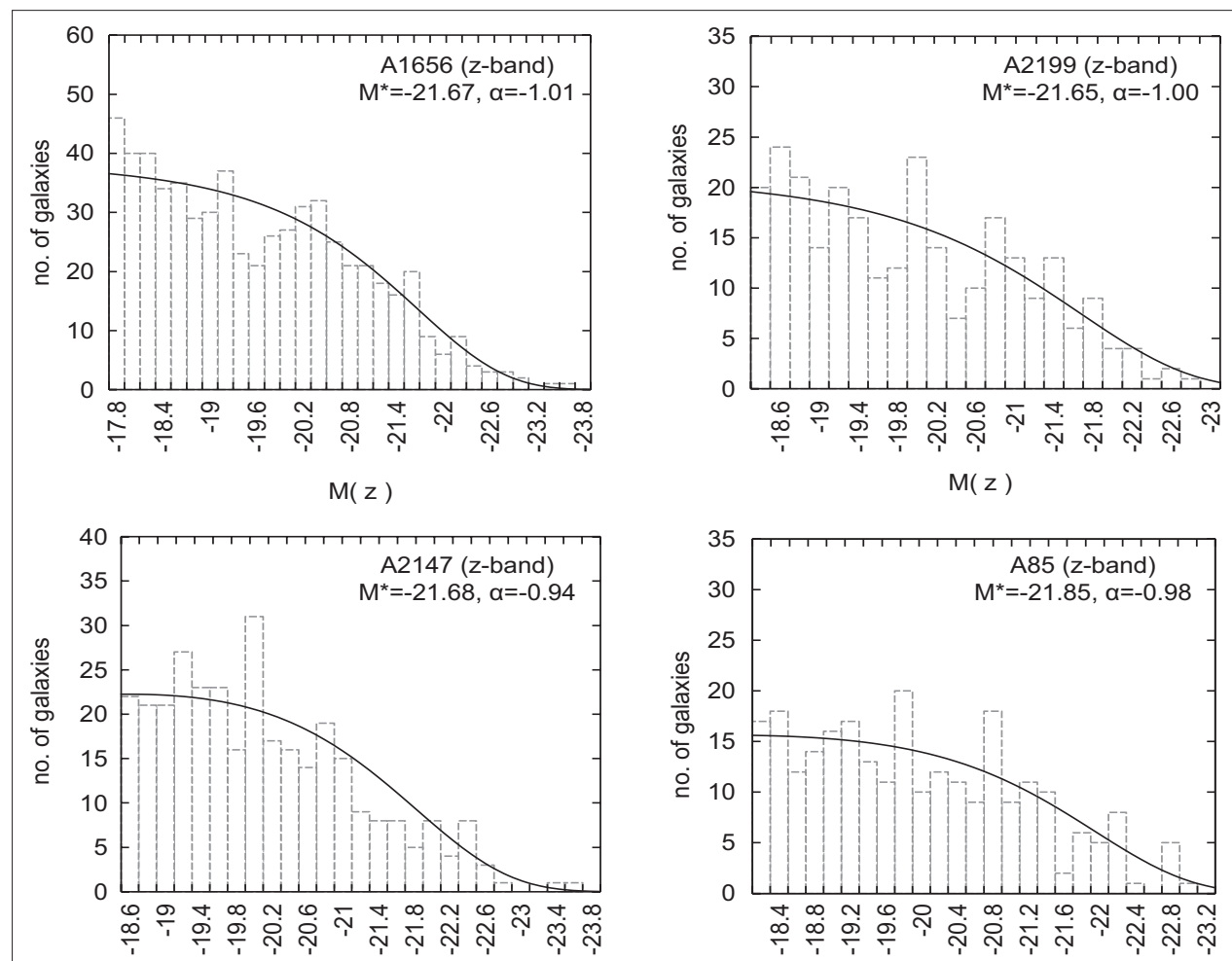

$\mathrm{M}(\mathrm{z})$

$\mathrm{M}(\mathrm{z})$
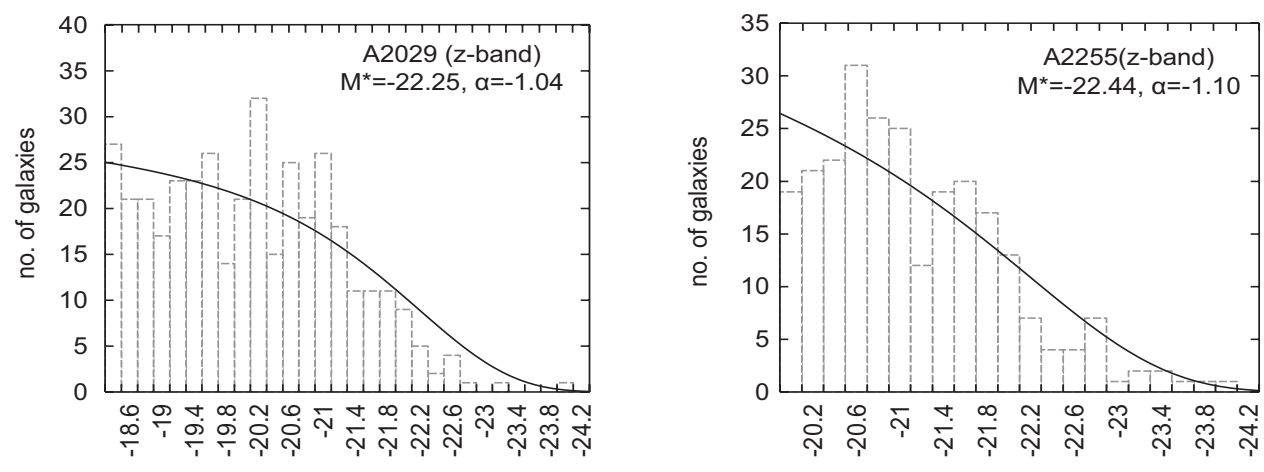

$\mathrm{M}(\mathrm{z})$

$M(z)$

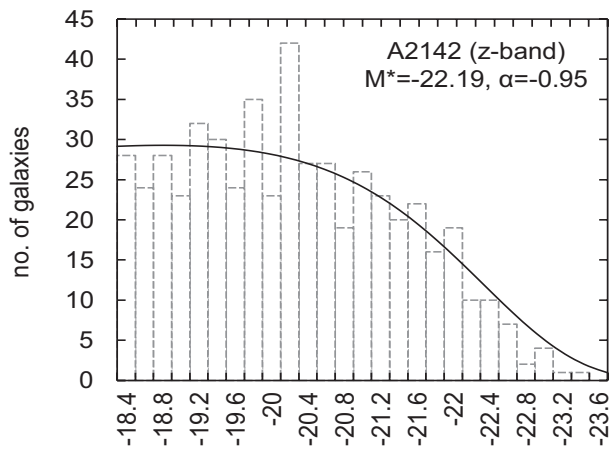

$\mathrm{M}(\mathrm{z})$

Fig. 5. The luminosity distributions (histograms) of the cluster sample in the z-band, fitted with Schechter functions (solid curves). 
the characteristic absolute magnitude, $\mathrm{M}^{*}$, and the faintend slope $\alpha$, as the shape of the LF is defined by these two parameters [29]. No remarkable variations are seen in both of these parameters, for all clusters, in each band, within the redshift range considered in this work. Furthermore, by noting the basic data listed in Table 1, we conclude that, in each band, the LF does not vary with such cluster characteristics as velocity dispersion (in agreement with Propis et al. [8]), richness (in agreement with Colless [6], Propis et al. [8]), and Bautz-Morgan morphology (in agreement with Colless [6], Propis et al. [8], Lugger [30]). The above results confirm the universality of the cluster LF, in agreement with several other works (for example, Colless [6]). For this reason, we can deal with the mean values of the Schechter parameters $\mathrm{M}^{*}$ and $\alpha$, for the whole clusters. These values are listed in Table 3 .

It is obvious from Table 3 that the characteristic absolute magnitude, $\mathrm{M}^{*}$, becomes brighter towards redder bands,

\section{TABLE 2: The best-fitting Schechter parameters for the cluster sample in the ugriz bands.}

\begin{tabular}{lcccccc}
\hline Cluster & $\begin{array}{c}\text { Schechter } \\
\text { parameters }\end{array}$ & u-band & g-band & r-band & i-band & z-band \\
\hline A1656 & $\phi^{*}$ & 5.19 & 4.46 & 4.69 & 5.28 & 5.40 \\
& $\mathrm{M}^{*}$ & -18.90 & -20.66 & -21.32 & -21.47 & -21.67 \\
& $\alpha$ & -1.12 & -1.10 & -1.08 & -1.04 & -1.01 \\
A2199 & $\phi^{*}$ & 5.03 & 3.54 & 3.15 & 2.76 & 2.99 \\
& $\mathrm{M}^{*}$ & -18.52 & -20.21 & -21.05 & -21.52 & -21.65 \\
& $\alpha$ & -0.86 & -1.00 & -1.03 & -1.06 & -1.00 \\
$\mathrm{~A} 2147$ & $\phi^{*}$ & 3.52 & 3.44 & 3.48 & 5.20 & 4.21 \\
& $\mathrm{M}^{*}$ & -18.92 & -20.64 & -21.31 & -21.27 & -21.68 \\
& $\alpha$ & -1.15 & -1.06 & -1.03 & -0.87 & -0.94 \\
$\mathrm{~A} 0085$ & $\phi^{*}$ & 3.19 & 2.63 & 2.42 & 2.35 & 2.61 \\
& $\mathrm{M}^{*}$ & -18.85 & -20.54 & -21.39 & -21.76 & -21.85 \\
& $\alpha$ & -1.00 & -1.01 & -1.02 & -1.02 & -0.98 \\
$\mathrm{~A} 2029$ & $\phi^{*}$ & 6.04 & 6.09 & 6.30 & 4.79 & 3.35 \\
& $\mathrm{M}^{*}$ & -18.66 & -20.16 & -20.80 & -21.38 & -22.25 \\
& $\alpha$ & -0.97 & -1.00 & -0.91 & -1.11 & -1.04 \\
$\mathrm{~A} 2255$ & $\phi^{*}$ & 7.09 & 3.81 & 5.07 & 2.36 & 3.52 \\
& $\mathrm{M}^{*}$ & -18.85 & -21.23 & -21.67 & -22.71 & -22.44 \\
& $\alpha$ & -0.75 & -1.10 & -0.91 & -1.22 & -1.10 \\
$\mathrm{~A} 2142$ & $\phi^{*}$ & 4.36 & 7.67 & 8.37 & 5.17 & 5.31 \\
& $\mathrm{M}^{*}$ & -19.46 & -20.43 & -21.03 & -21.90 & -22.19 \\
& $\alpha$ & -1.14 & -0.87 & -0.82 & -1.00 & -0.95 \\
\hline
\end{tabular}

\begin{tabular}{|c|c|c|}
\hline Band & $M *$ & $\alpha$ \\
\hline$u$ & $-18.88 \pm 0.11$ & $-1.00 \pm 0.06$ \\
\hline$g$ & $-20.55 \pm 0.13$ & $-1.02 \pm 0.03$ \\
\hline$r$ & $-21.22 \pm 0.11$ & $-0.97 \pm 0.04$ \\
\hline$i$ & $-21.72 \pm 0.18$ & $-1.05 \pm 0.04$ \\
\hline$z$ & $-21.96 \pm 0.12$ & $-1.00 \pm 0.02$ \\
\hline
\end{tabular}

while no remarkable change is noted in the value of the faint-end slope with passband. The reason for this variation of galaxy LF with passband is the contribution of different mechanisms in galaxy evolution. At ultraviolet, for example, the shape of the LF is strongly influenced by star formation since most of the flux is generated by young stars [31]. On the other hand, the LF in the red bands determines the typical stellar distribution [28]. The results in the present work are in good agreement with the previous works [32], [33]. The flat faint-end slope $(\alpha \sim-1)$ obtained in the present work (Table 3) agrees well with the one obtained by Blanton et al. (2003) [32]. This flat faint-end slope is a result of the disruption of a large number of dwarf galaxies inside clusters during the first stages of cluster formation [10]. At the bright end of the LF, the exponential decrease of the number density of galaxies is caused by various feedback processes quenching star formation in massive galaxies. The mechanisms proposed for this quenching are either the effect of supernova explosions or an accreting supermassive black hole. In either case, the gas content is heated and then ejected out of the galaxy, quenching star formation process.

\section{CONCLUSIONS}

The galaxy LFs of some nearby clusters were studied in all of the SDSS passbands ugriz. In each case, a Schechter function was fitted to the bright end of the distribution, using the $\chi^{2}-$ minimization technique, to obtain the best-fitting Schechter parameters, $\Phi^{*}, \mathrm{M}^{*}$, and $\alpha$. For each passband, no noticeable variations were observed in the values of $\mathrm{M}^{*}$ and $\alpha$ in any cluster. Further, it was found that the LF does not change with such cluster parameters as richness, velocity dispersion, and Bautz-Morgan morphology. From the mean values of $\mathrm{M}^{*}$ and $\alpha$, it was found that $\mathrm{M}^{*}$ becomes brighter toward redder bands, whereas no remarkable change was noted in the value of $\alpha$ with passband, being about $(-1.00)$.

\section{ACKNOWLEDGMENT}

Funding for SDSS-III has been provided by the Alfred P. Sloan Foundation, the Participating Institutions, the National Science Foundation, and the U.S. Department of Energy Office of Science. The SDSS-III web site is http://www. sdss3.org/.

SDSS-III is managed by the Astrophysical Research Consortium for the Participating Brazilian Participation Group, Brookhaven National Laboratory, Carnegie Mellon University, University of Florida, the French Participation 
Group, the German Participation Group, Harvard University, the Instituto de Astrofisica de Canarias, the Michigan State/ Notre Dame/JINA Participation Group, Johns Hopkins University, Lawrence Berkeley National Laboratory, Max Planck Institute for Astrophysics, Max Planck Institute for Extraterrestrial Physics, New Mexico State University, New York University, Ohio State University, Pennsylvania State University, University of Portsmouth, Princeton University, the Spanish Participation Group, University of Tokyo, University of Utah, Vanderbilt University, University of Virginia, University of Washington, and Yale University.

This research has made use of the NED which is operated by the JET propulsion Laboratory, California Institute of Technology, under contact with the National Aeronautics and Space Administration.

\section{REFERENCES}

[1] P. Schechter. "An analytic expression for the luminosity functions for galaxies. Astrophysical Journal, vol. 203, pp. 297-306, 1976.

[2] E. Hubble. "The luminosity function of nebulae. I. The luminosity function of resolved nebulae as indicated by their bright stars". Astrophysical Journal, vol. 84, p. 158, 1936.

[3] E. Hubble. "The luminosity function of nebulae. II. The luminosity function as indicated by residuals in velocity-magnitude relations". Astrophysical Journal, vol. 84, p. 270, 1936.

[4] F. Zwicky. "On the large scale distribution of matter in the universe". Physical Review, vol. 61, pp. 489-503, 1942.

[5] A. Oemler. "The systematic properties of clusters of galaxies. I. Photometry of 15 clusters". Astrophysical Journal, vol. 194, pp. 1-20, 1974.

[6] M. Colless. "The dynamics of rich clusters-II. Luminosity functions". Monthly Notices of the Royal Astronomical Society, vol. 237, pp. 799-826, 1989.

[7] E. J. Gaidos. "The galaxy luminosity function from observations of twenty Abell clusters". Astrophysical Journal, vol. 113, pp. 117-129, 1997.

[8] R. De Propis, M. Colless, S. P. Driver, W. Couch, J. A. Peacock, I. K. Baldry, C. M. Baugh, J. Bland-Hawthorn, T. Bridges, R. Cannon, S. Cole, C. Collins, N. Cross, G. B. Dalton, G. Efstathiou, R. S. Ellis, C. S. Frenk, K. Glazebrook, E. Hawkins, C. Jackson, O. Lahav, I. Lewis, S. Lumsden, S. Maddox, D. S. Madgwick, P. Norberg, W. Percival, B. Peterson, W. Sutherland and K. Taylor. "The 2dF galaxy redshift survey: The luminosity function of cluster galaxies". Monthly Notices of the Royal Astronomical Society, vol. 342, pp. 725, 2003.

[9] A. Dressler. "A comprehensive study of 12 very rich clusters of galaxies. I. Photometric technique and analysis of the luminosity function". Astrophysical Journal, vol. 223, pp. 765-787, 1978.

[10] O. López-Cruz, H. K. C. Yee, J. P. Brown, C. Jones and W. Forman. "Are luminous CD halos formed by the disruption of dwarf galaxies"? ApJ, vol. 475, p. L97, 1997.

[11] P. Popesso, A. Biviano, H. Böhringer and M. Romaniello. "RASSSDSS galaxy cluster survey. IV. A ubiquitous dwarf galaxy population in clusters". Astronomy Astrophysics, vol. 445, pp. 2942, 2006.

[12] R. De Propris, P. R. Eisenhardt, S. A. Stanford and M. Dickinson. "The infrared luminosity function of galaxies in the Coma cluster". Astrophysical Journal, vol. 503, p. L45, 1998.

[13] L. Cortese, G. Gavazzi, A. Boselli, J. Iglesias-Paramo, J. Donas and B. Milliard. "The UV luminosity function of nearby clusters of galaxies". Astronomy Astrophysics, vol. 410, p. L25, 2003.

[14] L. Bai, G. H. Rieke, M. J. Rieke, J. L. Hinz, D. M. Kelly and M. Blaylock. "Infrared luminosity function of the Coma cluster". Astrophysical Journal, vol. 639, pp. 827, 2006.

[15] C. A. Valotto, M. A. Nicotra, H. Muriel and D. G. Lambas. "The luminosity function of galaxies in clusters". Astrophysical Journal, vol. 479, p. 90, 1997.

[16] M. Yagi, N. Kashikawa, M. Sekiguchi, M. Doi, N. Yasuda, K. Shimasaku and S. Okamura. Luminosity functions of 10 nearby clusters of galaxies. II. Analysis of the luminosity function". Astrophysical Journal, vol. 123, p. 87, 2002.

[17] S. M. Hansen, T. A. McKay, R. H. Wechsler, J. Annis, E. S. Sheldon and A. Kimball. "Measurement of galaxy cluster sizes, radial profiles, and luminosity functions from SDSS photometric data". Astrophysical Journal, vol. 633, p. 122, 2005.

[18] B. Binggeli, A. Sandage and G. A. Tammann. "The luminosity function of galaxies". Annual Review of Astronomy and Astrophysics, vol. 26, pp. 509-560, 1988.

[19] A. Dressler. "Galaxy morphology in rich clusters: Implications for the formation and evolution of galaxies". Astrophysical Journal, vol. 236, pp. 351-356, 1980.

[20] G. O. Abell, H. G. Corwin and R. P. Olowin. "A catalog of rich clusters of galaxies". Astrophysical Journal Supplement Series, vol. 70, p. 1, 1989.

[21] C. P. Ahn, R. Alexandroff, C. A. Prieto, S. F. Anderson, T. Anderton, B. H. Andrews, É. Aubourg, S. Bailey, E. Balbinot, and R. Barnes. "The ninth data release of the sloan digital sky survey: First spectroscopic data from the SDSS-III baryon oscillation spectroscopic survey". Astrophysical Journal Supplement Series, vol. 203, p. 21, 2012.

[22] I. Agulli, J. A. L. Aguerri, R. Sánchez-Janssen, C. Dalla Vecchia, A. Diaferio, R. Barrena, L. Dominguez Palmero and H. Yu. "Deep spectroscopy of nearby galaxy clusters I. Spectroscopic luminosity function of Abell 85". Monthly Notices of the Royal Astronomical Society, vol. 458, p. 1590-1603, 2016.

[23] J. Sohn, M. J. Geller, H. J. Zahid, D. G. Fabricant and A. Diaferio. "The velocity dispersion function of very massive galaxy clusters: Abell 2029 and Coma". Astrophysical Journal Supplement Series, vol. 229, p. 20, 2017.

[24] E. F. Schlafly and D. P. Finkbeiner. "Measuring reddening with Sloan Digital Sky Survey stellar spectra and recalibrating SFD". Astrophysical Journal, vol. 737, p. 103, 2011.

[25] I. V. Chilingarian, A. L. Malchoir and I. Y. Zolotukin. "Analytical approximations of K-corrections in optical and near-infrared bands". Monthly Notices of the Royal Astronomical Society, vol. 405, pp. 1409-1420, 2010.

[26] I. Chilingarian and I. Zoloyukin. "A universal ultraviolet-optical colour-colour-magnitude relation of galaxies". Monthly Notices of the Royal Astronomical Society, vol. 419, pp. 1727-1739, 2012.

[27] M. S. Longair. Galaxy Formation. Springer, Germany, 2008.

[28] P. Schneider. Extragalactic Astronomy and Cosmology: An Introduction, Springer, Germany, 2006. 
[29] H. Karttunen, P. Kröger, H. Oja, M. Poutanen and K. J. Donner. Fundamental Astronomy. Springer, Germany, 2007.

[30] P. M. Lugger. Luminosity functions for nine Abell clusters". Astrophysical Journal, vol. 303, pp. 535-555, 1986.

[31] R. De Propris, M. Bremer and S. Phillips. "Luminosity functions of cluster galaxies. The near-ultraviolet luminosity function at $\langle z\rangle \sim$ 0.05". Astronomy Astrophysics, vol. 1807, p. 10775, 2018.
[32] M. R. Blanton, J. Brinkmann, I. Csabai, M. Doi, D. Eisenstein, M. Fukugita, J. E. Gunn, D. W. Hogg and D. J. Schlegel. "Estimating fixed-frame galaxy magnitudes in the Sloan Digital Sky Survey". Astronomical Journal, vol. 125, pp. 2348-2360, 2003.

[33] P. Popesso, H. Böhringer, M. Romaniello and W. Voges. "RASSSDSS galaxy cluster survey. II. A unified picture of the cluster luminosity function". Astronomy Astrophysics, vol. 433, pp. 415429, 2005. 